\title{
Passivity Controller Based on Load-Side Damping Assignment for High Stiffness Controlled Series Elastic Actuators
}

\author{
Hyunwook Lee ${ }^{(0)}$, Student Member, IEEE, Jee-Hwan Ryu ${ }^{(0)}$, Senior Member, IEEE, \\ Jinoh Lee (1), Senior Member, IEEE, and Sehoon Oh (), Senior Member, IEEE
}

\begin{abstract}
This article offers a new insight to the achievable stiffness limitation of the impedance control of series elastic actuators (SEAs) by suggesting a comparative analysis of energy ports and passivity control framework at the energy port to enhance the maximum achievable rendered stiffness of SEAs. To this end, it is explored that SEAs have two different port definitions to assess energy and passivity of the system-spring port and load port; and conservatisms for passivity evaluation of two ports are investigated and compared utilizing frequency characteristics. The results reveal that the load port passivity exhibits less conservatism, which allows to render larger achievable stiffness in impedance-controlled SEAs. Moreover, key parameters that determine the passivity characteristic of the SEA impedance control are discovered based on the load port passivity analysis. A novel passivity control framework that incorporates the time-domain passivity observer and the passivity controller is designed utilizing the load port energy monitoring, which offers a less conservative assessment of the systems passivity. Throughout this novel port passivity analysis and the passivity control, it can be achieved to render maximum rendered stiffness of the SEA impedance control much higher than the limitation that has been perceived as the maximum value.
\end{abstract}

Index Terms-Force control, impedance, stability analysis.

\section{INTRODUCTION}

$\mathbf{S}$ AFETY has to be taken into account when the robots achieve the tasks for human-robot interaction and

Manuscript received November 30, 2019; revised April 10, 2020 and June 19, 2020; accepted July 14, 2020. Date of publication August 7, 2020; date of current version October 19, 2020. This work was supported by the National Retail Federation by the Korea government (NRF2019R1A2C2011444 and NRF-2020RIA2C2004169). (Corresponding author: Sehoon Oh.)

Hyunwook Lee and Sehoon Oh are with the Department of Robotics Engineering, DGIST, Daegu 42988, South Korea (e-mail: hwlee@dgist.ac.kr; sehoon@dgist.ac.kr).

Jee-Hwan Ryu is with the Department of Civil and Environmental Engineering, KAIST, Daejeon 34141, South Korea (e-mail: jhryu@kaist.ac.kr).

Jinoh Lee was with the Advanced Robotics Department, Istituto Italiano di Tecnologia (IIT), 16163 Genoa, Italy. He is now with the Institute of Robotics and Mechatronics Center, German Aerospace Center (DLR), 82234 Weßling, Germany (e-mail: jnoh.lee@dlr.de).

Color versions of one or more of the figures in this article are available online at https://ieeexplore.ieee.org.

Digital Object Identifier 10.1109/TIE.2020.3013751 collaboration, where there is a particular need to develop actuation systems that can measure forces and to achieve compliant behaviors. Series elastic actuator (SEA), originally introduced in [1], has been established as a potential solution, since it has an intrinsic elasticity due to a spring element, which allows accurate force sensing and shock reduction capabilities. Accordingly, SEA is utilized to attain safe yet high force capacity, and a variety of controllers has been devised to improve its performance [2]-[6].

An impedance control is widely employed for SEA thanks to its high force control performance, and impedance-controlled SEAs have been used in a variety of applications such as humanoids (e.g., Valkyrie [7], [8], THOR [9], and CoMan [10]), and exoskeletons [11], [12]. Impedance control should be able to achieve high stiffness rendering in addition to lowering impedance.

For example, it is needed to prevent swaying behaviors in robot walking, or to stiffen interaction responses to human for a physical exercise application [13]. On top of this wide impedance rendering ability, it is also essential for the impedance control to ensure the stability of the SEAs when they interact with various environments including humans. For control systems contacting with environments to ensure stability, passivity concept has been mostly used [14]. Passivity is a sufficient condition to guarantee coupled stability when it contacts to external environments, since the system coupled with the passive environment results in the stability of the whole system. Moreover, passivity can be measured using only the interacting signals at one port (without any system parameters) while it still can tell the stability of the whole coupled system.

Correspondingly, the passivity has become a popular tool to analyze the stability, particularly for applications interacting with unknown passive environments such as haptic and telerobotic systems.

However, there is a significant constraint in the impedancecontrolled SEA, which has been investigated in lots of literature [15]-[18]: "the maximum achievable stiffness must be limited lower than the inherent stiffness of the spring to guarantee the passivity of SEA." Transfer function of impedance is utilized to investigate the passivity in the frequency domain. Throughout this analysis, it is revealed that the passivity is violated when the desired stiffness in the impedance control is assigned to exceed the inherent physical spring stiffness of the SEA. 
Vallery et al., [15] first discussed the limitation of rendered stiffness concerning the passivity condition of the impedancecontrolled SEA, where the passivity analysis is conducted based on energy variables such as the load velocity and the spring force in SEA.

Tagliamonte and Accoto [16] also showed passivity at the spring port is violated when implementing higher viscoelasticity than physical impedance. In [17], since it was not successful to exceed spring stiffness even with several controllers such as velocity-sourced impedance control and admittance control, the authors, instead, proposed acceleration-based control to overcome the limitation, which enhances control performance of an inner-loop force controller. Nevertheless, increasing stiffness is hindered by limited force controller performance. Since the maximum achievable stiffness is a significant restriction in impedance control of SEA, the term "K-width was coined in [18] as the ratio of the achievable rendered stiffness to the physical spring stiffness as criteria to evaluate the performance of the SEA impedance control. They also investigated how discretization, i.e., sampling frequency, affects the passivity of the impedancecontrolled SEA utilizing K-width, it was found that the K-width cannot become over 1 but sometimes it becomes less than 1 . Nevertheless, of the aforementioned aspects, this article underlines that it is practically achievable to increase active stiffness in the impedance-controlled SEA higher than the spring stiffness. Recently, it is found in [19] that this conservatism is stemmed from the energy port definition in passivity analysis, which is described at the spring port, while the passivity condition can be relaxed when the energy dissipation is analyzed at the load port, taking into consideration the precise load dynamics.

However, the approach in [19] relies only on the inherent damping of the SEA; there are still demands for further investigation of other influencing factors and extended energy control method to preserve the passivity in real-time, accordingly. More specifically, the effect of load-side damping can be exploited and controlled to raise the level of the maximum achievable stiffness.

Therefore, this article proposes a new controller to enhance the passivity of SEA, which can accommodate the load-side damping under appropriate realtime monitoring of system's energy.

For energy-based control, passivity controller (PC) strategy [20]-[22] has been employed to enhance the passivity of a system by adaptively dissipating the energy in real-time. This approach measures the energy of the system and absorbs the net energy when the passivity of the system is violated. In [23], the PC was applied to the impedance-controlled SEA, where the conventional time-domain passivity approach (TDPA) was modified for SEA by adjusting the desired impedance; when the passivity observer (PO) detects the passivity violation, then the modified PC reduces the desired stiffness of impedance control to recover the passivity. However, this method sacrifices the achievable stiffness level of SEA to recover the passivity condition, which is a deterioration in terms of high impedance control. Whereas, this article proposes a new strategy for the PC, which can achieve better impedance control of SEAs by modifying the load damping directly at the load port, where the passivity can be recovered without sacrificing the high stiffness
TABLE I

SUMmaRY OF RESEARCH ON PASSIVITY BASED APPROACH OF IMPEDANCE-CONTROLLED SEA

\begin{tabular}{ccccc}
\hline \hline Paper & $\begin{array}{c}\text { Impedance } \\
\text { controller }\end{array}$ & $\begin{array}{c}\text { Energy } \\
\text { port }\end{array}$ & $\begin{array}{c}\text { Achievable stiffness } \\
\text { under passivity }\end{array}$ & $\begin{array}{c}\text { Passivity } \\
\text { controller }\end{array}$ \\
\hline$[15]$ & Pure stiffness & Spring & $K_{\text {des }}<K$ & No control \\
\hline$[16]$ & Viscoelasticity & Spring & $K_{\text {des }}<K$ & No control \\
\hline$[17]$ & $\begin{array}{c}\text { Various inner } \\
\text { controllers }\end{array}$ & Spring & $K_{\text {des }}<K$ & No control \\
& $\begin{array}{c}\text { Acceleration } \\
\text { controller }\end{array}$ & Spring & $K_{\text {des }}>K$ & No control \\
& Pure stiffness & Spring & $K_{\text {des }}<K$ & No control \\
\hline$[18]$ & Pure stiffness & Load & $K_{\text {des }}>K$ & No control \\
\hline$[19]$ & Ppring & $K_{\text {des }}>K$ & $\begin{array}{c}\text { Stiffness } \\
\text { adjustment }\end{array}$ \\
\hline$[23]$ & Pure stiffness & Spartially) & $\begin{array}{c}\text { Damping } \\
\text { injection }\end{array}$ \\
\hline This & Pure stiffness & Load & $K_{\text {des }} \gg K$ & \\
paper & & & & \\
\hline \hline
\end{tabular}

in all situations, by taking advantage of the load port energy analysis.

Table I provides an overview of passivity-based approaches for the impedance-controlled SEA, where the major differences are given by the definition of the energy port at which the passivity analysis is conducted and the type of controller to make the passivity recovered when it is violated. Unlike other existing approaches, this article proposes a method to alleviate the conservative passivity condition by revisiting the energy port analysis for the SEA; a novel PC algorithm is derived to increase the achievable active stiffness much higher than the physical one in the SEA, i.e., the conventional theoretical limit, which has been one of the hurdles in passivity-based control. As a result, the highest stiffness can be achieved among the works related to passivity analysis of the impedance-controlled SEA.

Taking these issues on passivity and impedance control of SEAs into consideration, this article strives to make the following contributions.

1) The well-known passivity limitation of the impedancecontrolled SEA is revisited and mathematically analyzed considering the location of energy ports; the advantage of the load port is thus discussed over the spring port.

2) In addition to the comparative analysis between the passivity at the load port and the passivity at the spring port, a parametric analysis is performed to investigate key parameters for preserving passivity taking advantage of the load-port passivity analysis.

3) Noncollocated approach, which is different from conventional TDPA, is proposed. PO is designed to monitor energy at the load port, and a new PC is proposed that can modify the load-side damping in order to relax the passivity condition at the load port. Accordingly, it can maximize the achievable rendered stiffness over the physical stiffness level of the spring to which numerous former studies set the limit.

\section{PAssivity AnAlysis of SEAS}

This section investigates the passivity of impedancecontrolled SEA at two different energy ports, which are defined based on the SEA dynamic model. 


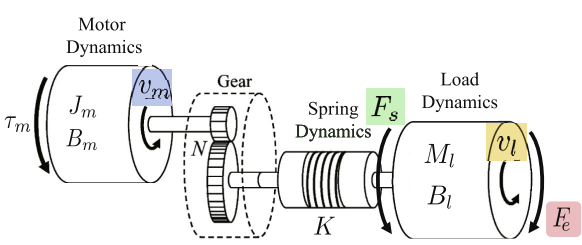

Fig. 1. Schematic diagram of the SEA.

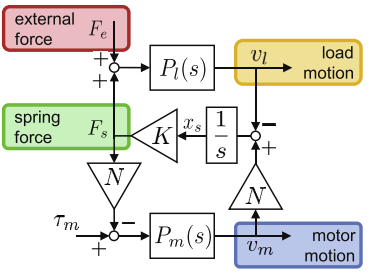

(a)

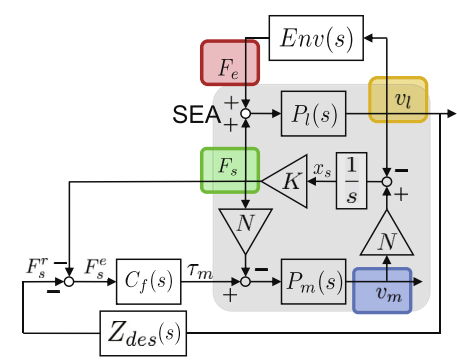

(b)
Fig. 2. Block diagram. (a) SEA. (b) Impedance-controlled SEA system. The environment interacts with the SEA through the external force $F_{e}$. The impedance controller $Z_{\text {des }}$ and inner force controller $C_{f}$ are applied. $F_{s}^{r}$ and $F_{s}^{e}$ indicate reference and error of spring force.

\section{A. Dynamics of SEAs With Impedance Control}

SEAs can be modeled as two rigid parts interconnected by a spring. Fig. 1 illustrates the schematics of the SEA dynamics, which includes a motor, a spring, and a load part. Motor torque $\tau_{m}$ rotates motor inertia $J_{m}$ and generates the angular motor position $\theta_{m}$, which is affected by motor viscous friction $B_{m}$. The reduction ratio of a gear is denoted as $N$.

The load part consists of its mass $M_{l}$ and damping $B_{l}$, and its position is given as $x_{l}$. The spring part generates the spring force $F_{s}$ where $K$ is stiffness, and the spring force is transmitted to the load and the motor.

The SEA dynamics can be expressed in the time domain as follows:

$$
\begin{aligned}
M_{l} \ddot{x}_{l}+B_{l} \dot{x}_{l} & =F_{s}+F_{e} \\
J_{m} \ddot{\theta}_{m}+B_{m} \dot{\theta}_{m}+N F_{s} & =\tau_{m} \\
F_{s} & =K\left(N \theta_{m}-x_{l}\right)
\end{aligned}
$$

where $F_{e}$ is the external force applied from the environment. The spring force $F_{s}$ in (3) is transmitted to the motor-side dynamics in (2) and the load-side dynamics in (1). The dynamics of the SEA also can be expressed in the $S$-domain and represented as the block diagram illustrated in Fig. 2(a). The motor dynamics $P_{m}(s)$ and the load dynamics $P_{l}(s)$ in Fig. 2(a) are expressed in the $S$-domain as follows:

$$
P_{m}(s)=\frac{1}{J_{m} s+B_{m}}, P_{l}(s)=\frac{1}{M_{l} s+B_{l}} .
$$

Based on the derived SEA dynamics, the impedance control of the SEA is constructed as in Fig. 2(b), where the force controller $C_{f}$ is configured in the inner loop, and the desired impedance $Z_{\text {des }}$ envelops the force control in the outer loop. The impedance can be rendered in another way, namely, the admittance control,

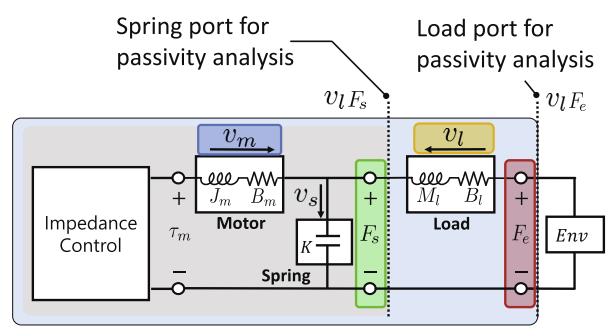

Fig. 3. Port definition of SEA for passivity analysis. The beige area represents spring port and the azure area represents load port.

where the position is controlled with respect to the measured force. However, the impedance control shown in Fig. 2(b) has been widely employed and studied for SEA due to its high force control performance [15]-[18], [24], whereas the admittance control is rarely used in the SEA [17], [25].

Among various force controllers, a simple proportionalderivative (PD) control is implemented which is commonly used for passivity analysis in impedance control [26]; the force control $C_{f}$ is thus expressed as follows: ${ }^{1}$

$$
C_{f}=\Lambda_{p}+\Lambda_{d} s
$$

where $\Lambda_{p}$ and $\Lambda_{d}$ denote the proportional (P)- and derivative (D)-gains, respectively. The desired impedance $Z_{\text {des }}$ is designed as follows:

$$
Z_{\mathrm{des}}=M_{\mathrm{des}} s+D_{\mathrm{des}}+\frac{K_{\mathrm{des}}}{s}
$$

where $M_{\mathrm{des}}, D_{\mathrm{des}}$, and $K_{\mathrm{des}}$ are the desired mass, damping, and stiffness of impedance, respectively. It is true that $M_{\mathrm{des}}$ and $D_{\text {des }}$ are the impedance parameters to be designed properly; however, this article focuses on the design of $K_{\text {des }}$ because it is the most critical factor causing the passivity violation. It is also noticeable that the passivity is violated when the large desired stiffness is set, whereas the increasing desired mass and damping does not make the violation.

The impedance-controlled SEA can also be described as network system consisting of the dynamics, the controller and the environment, where each part interacts with others through energy transfer as in Fig. 3.

This network description clarifies two energy interaction ports in SEA: one port is defined between the spring and the load (spring port), the other port is defined between the load and the environment (load port).

\section{B. Passivity Analysis Depending on Energy Ports}

The passivity of the linear systems can be analyzed at the frequency domain by checking the phase characteristic of the controlled impedance. When the phase of the impedance deviated from the range of $-90^{\circ} \sim 90^{\circ}$, then the system becomes nonpassive [14]. Taking this point into consideration, the passivity conditions at two ports can be compared by the phase characteristic analysis of the transfer functions at two ports.

\footnotetext{
${ }^{1} \mathrm{PD}$ control is selected based on its wide popularity in practical engineering, readers can thus benefit from analysis results in this article. Moreover, the passivity analysis and newly proposed passivity controller can be extended to any other controllers, not limited to PD control.
} 


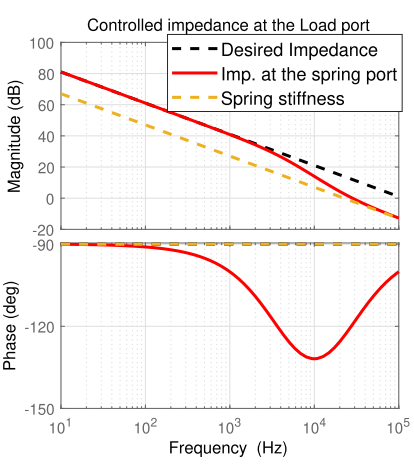

(a)

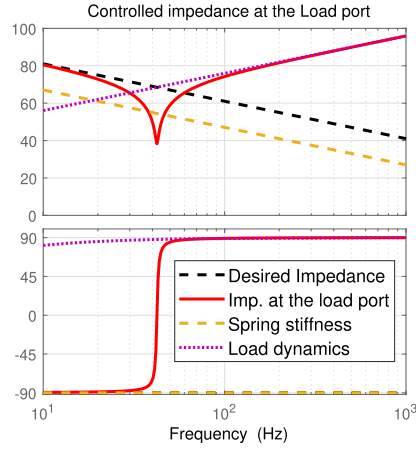

(b)
Fig. 4. Comparison of desired and controlled impedances. (a) Spring port $Z_{s}$ (from load velocity $v_{l}$ to spring force $F_{s}$ ). (b) Load port $Z_{l}\left(v_{l}\right.$ to external force $F_{e}$ ). Desired impedance $K_{\text {des }}$ is $5 K$.

TABLE II

PARAMETERS UtILIZED IN THE SiMULATION

\begin{tabular}{cll}
\hline \hline Parameter & Description & Value \\
\hline$J_{m}$ & Motor inertia & $0.64 \mathrm{e}-5 \mathrm{~kg} \cdot \mathrm{m}^{2}$ \\
$B_{m}$ & Motor damping & $0.6 \mathrm{e}-4 \mathrm{Nm} \cdot \mathrm{s} / \mathrm{rad}$ \\
$K$ & Spring stiffness & $160 \mathrm{kN} / \mathrm{m}$ \\
$N^{-1}$ & Gear ratio & 7854 \\
$M_{l}$ & Load mass & $2 \mathrm{~kg}$ \\
$B_{l}$ & Load damping & $0 \sim 200 \mathrm{~N} \cdot \mathrm{s} / \mathrm{m}$ \\
$\Lambda_{p}$ & Inner force P gain & $0 \sim 100$ \\
$\Lambda_{d}$ & Inner force D gain & $0 \sim 0.01$ \\
\hline \hline
\end{tabular}

The controlled impedance at spring port can be derived from the load velocity $v_{l}$ to the spring force $F_{s}$, given as follows:

$$
Z_{s}(s)=\frac{F_{s}}{v_{l}}=\frac{N K Z_{\mathrm{des}} P_{m} C_{f}+K}{N K P_{m} C_{f}+N^{2} K P_{m}+s} .
$$

From (7), the frequency characteristics of the controlled impedance at the spring port $Z_{s}(s)$ can be illustrated as Fig. 4(a), where the desired stiffness $K_{\text {des }}$ is set larger than the spring stiffness $K\left(K_{\text {des }}=5 K\right)$. The other parameters utilized in the simulation are listed in Table II.

In Fig. 4(a), the phase of the controlled impedance drops below $-90^{\circ}$, which indicates that the system is nonpassive.

Similarly, the passivity of the SEA at the load port can be analyzed by using the impedance transfer function $Z_{l}(s)$ from the load velocity $v_{l}$ to the external force $F_{e}$, derived as follows:

$$
\begin{aligned}
Z_{l}(s)=\frac{F_{e}}{v_{l}} & =M_{l} s+B_{l}+\frac{N K Z_{\mathrm{des}} P_{m} C_{f}+K}{N K P_{m} C_{f}+N^{2} K P_{m}+s} \\
& =M_{l} s+B_{l}+Z_{s}(s) .
\end{aligned}
$$

Fig. 4(b) shows the frequency characteristics of the controlled impedance at the load port $Z_{l}(s)$, where the parameters are set to the same values as Fig. 4(a).

The phase of the controlled impedance at the load port remains within $-90^{\circ} \sim 90^{\circ}$. The contrast between Fig. 4(a) and (b) implies that the characteristics of phase changes in large amounts according to the energy ports, which leads to the conclusion that the passivity analysis at the load port is less conservative than the analysis at the spring port.

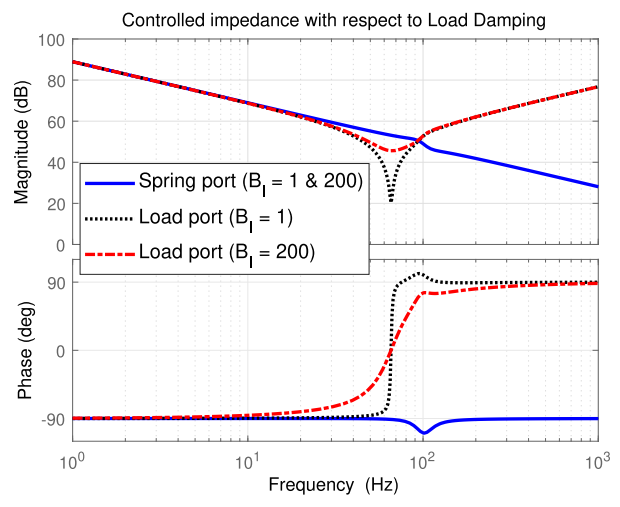

Fig. 5. Frequency characteristics of the controlled impedance with respect to the load damping.

\section{Analysis of Passivity Condition at Load Port Using Frequency Characteristics}

By taking advantage of the less conservative passivity analysis at the load port, it is investigated how the passivity condition of SEA at the load port changes with respect to several different load and control parameters.

Fig. 5 compares the frequency characteristics of the SEA at the load port and the spring port with several different load damping values $B_{l}$, when the SEA is impedance controlled to have a stiffness higher than the inherent stiffness $\left(K_{\text {des }}=1.1 K\right)$. The P-gain $\Lambda_{p}$ and D-gain $\Lambda_{d}$ for force control are set to 0.12 and $1 \mathrm{e}-5$, respectively. The result reveals that the phase condition at the load port can be recovered to remain within $-90^{\circ} \sim 90^{\circ}$ as $B_{l}$ increases, and thus the system becomes passive.

Notice that the phase characteristics do not change at the spring port even with the variation of $B_{l}$, which means the passivity cannot be recovered at the spring port.

The effect of the force feedback gains on the passivity can be investigated utilizing the frequency characteristic at the load port. Fig. 6 shows how the frequency responses change with respect to the different force feedback gains $\Lambda_{d}$ 's [in Fig. 6(a)] and $\Lambda_{p}$ 's [in Fig. 6(b)]. Fig. 6(a) reveals that the D-gain $\left(\Lambda_{d}\right)$ improves the passivity condition at the load port. Namely, too small D-gain can deteriorate the passivity condition of SEA leading to a phase surge at a certain frequency where the phase most deviates, while large D-gain can restore the phase at the certain frequency relaxing the passivity condition at the load port. Notice that the passivity at the spring port is still violated regardless of whether D-gain is large enough or not. Fig. 6(b) displays that P-gain affects the location of the phase-deviating frequency rather than the phase characteristics around it. Therefore, the passivity of the SEA at the load port cannot be recovered by increasing $\Lambda_{p}$.

\section{Effect of Load Damping and D-Gain on Maximum Achievable Stiffness}

It is verified that the load damping and the $\mathrm{D}$-gain can improve the passivity condition of the SEA in Section II-C, particularly when it is controlled to exhibit high impedance. The following question will be, how the limitation of the achievable stiffness 


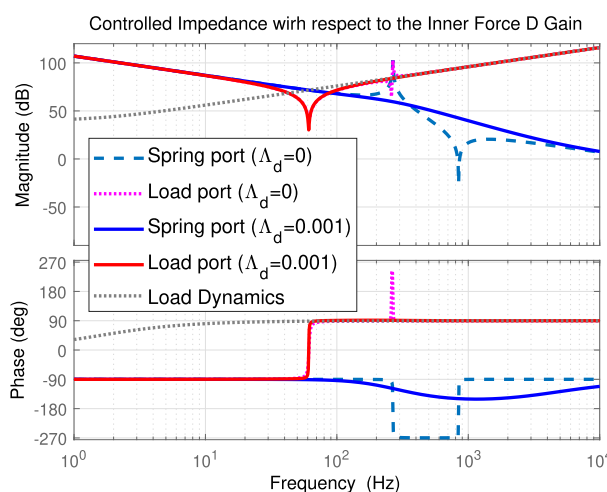

(a)

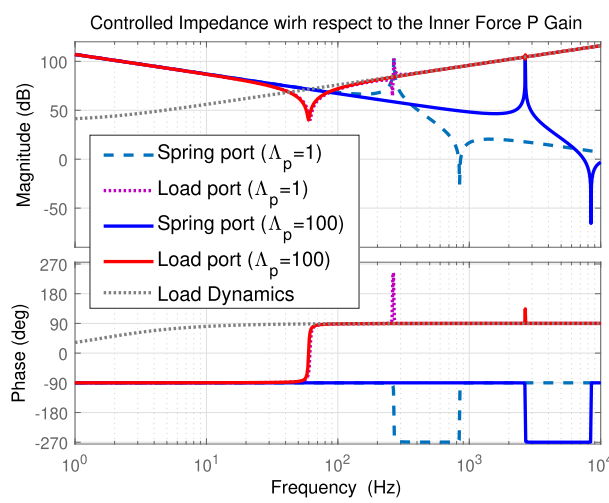

(b)

Fig. 6. Frequency characteristics of the controlled impedance with respect to (a) inner force D-gain (b) inner force P-gain.

is determined when the load damping and the feedback gains are given. Therefore, this section elaborates on the relationship among the achievable stiffness, the load damping and the force control gains.

The SEA system is passive when the impedance in (8) is positive real, which means the real part of the derived impedance should be positive [14]. Hence, the condition for the passivity of SEA can be derived as follows:

$$
\begin{aligned}
& \Re\left(Z_{l}(i \omega)\right) \\
& =\frac{N B_{m} K\left(N K-K_{\mathrm{des}} \Lambda_{p}+K \Lambda_{p}\right)}{\left(B_{m} \omega+N K \Lambda_{d} \omega\right)^{2}+\left(N K \Lambda_{p}+N^{2} K-J_{m} \omega^{2}\right)^{2}} \\
& \quad+\frac{N K \Lambda_{d}\left(J_{m} K \omega^{2}-J_{m} K_{\mathrm{des}} \omega^{2}+K_{\mathrm{des}} N^{2} K\right)}{\left(B_{m} \omega+N K \Lambda_{d} \omega\right)^{2}+\left(N K \Lambda_{p}+N^{2} K-J_{m} \omega^{2}\right)^{2}} \\
& \quad+B_{l}>0 . \quad(\forall \omega \geq 0) .
\end{aligned}
$$

Based on this inequality, the maximum achievable stiffness $K_{\text {des }}$ can be calculated when a set of parameters $B_{l}, \Lambda_{p}$, and $\Lambda_{d}$ is given.

For better evaluation of the achievable stiffness, the KWidth [18] represented as $n=K_{\text {des }} / K$, is utilized as the criteria to evaluate the maximum stiffness the SEA impedance control can achieve. Fig. 7 describes the relationship between $n$ and other parameters. At first, the maximum achievable $n$ with respect to the P-gain $\Lambda_{p}$ and the load damping $B_{l}$ is plotted in

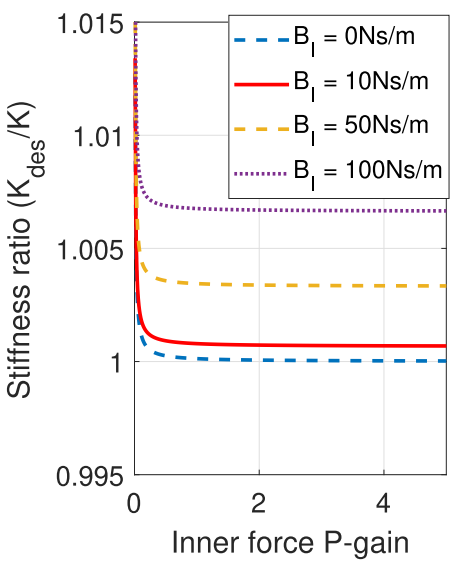

(a)

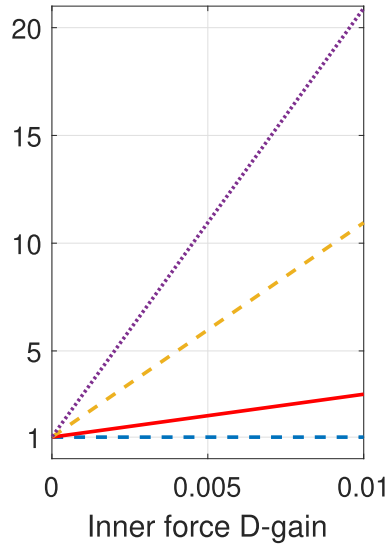

(b)
Fig. 7. Stiffness ratio $n$ of the spring stiffness to the achievable stiffness. (a) Inner force P-gain $\Lambda_{p}$. (b) D-gain $\Lambda_{d}$.

TABLE III

SUMMARY OF SECTION II

\begin{tabular}{clc}
\hline \hline Energy port & Parameter & Passivity constraint \\
\hline Spring port & Inner force PD-gains & $K_{\text {des }}<K$ \\
& Load damping & $K_{\text {des }}<K$ \\
\hline Load port & Inner force PD-gains & $K_{\text {des }}<K$ \\
& Load damping & $K_{\text {des }}>K$ \\
& D-gain + Load damping & $K_{\text {des }} \gg K$ \\
\hline \hline
\end{tabular}

Fig. 7(a). The result shows that the load damping can increase the maximum stiffness while the $\mathrm{P}$ gain cannot affect it.

The K-Width $n$ with respect to the D-gain $\Lambda_{d}$ and the load damping is investigated in Fig. 7(b), which verifies that the Dgain can increase the K-Width $n$, and the load damping also affects $n$. In particular, the larger the load damping value is the greater the influence of the D gain on the increase in the maximum achievable stiffness.

Table III summarizes the investigation in Section II, which draws a conclusion that the passivity condition of the SEA can be relieved by employing the load port, and the maximum achievable stiffness can be increased by appropriate design of the load damping and force feedback derivative gain.

\section{PO AND PC FOR SEA}

Although, it is essential to confirm that load damping and controller parameters can increase the achievable stiffness of the impedance-controlled SEA,

it is difficult to tell whether the desired stiffness violates the passivity or not in real applications. If there is a way to monitor the passivity condition in real-time during operations, it can be constantly evaluated whether the desired stiffness violates the passivity or not. Then, the passivity-related parameters can be adjusted accordingly.

\section{A. $P O$ and $P C$}

TDPA [20]-[22] has been used for guaranteeing the passivity of haptic and telerobotic systems. It is composed of two components: $\mathrm{PO}$ is an energy monitoring component for observing 
passivity in real-time, and PC exploits this PO and modifies the control input to recover passivity only when it is necessary.

The passivity of the one port network is defined at the time domain as follows [20]:

Definition: The one-port network with initial energy storage $E(0)$ is passive if and only if

$$
\int_{0}^{t} f(\tau) v(\tau) d \tau+E(0) \geq 0, \quad \forall t \geq 0
$$

where $E(0)$ is the initial energy storage, $f$ is the force and $v$ is the velocity at the port. Equation (10) implies that the net energy supplied into the network should be greater than the negative of initial energy storage $(E(0))$ to be passive.

As mentioned in Section II, the passivity can be defined for SEA based at two different energy ports, and thus

POs can be designed at both ports as follows:

$$
\hat{E}_{s}=\int_{0}^{t} \hat{F}_{s}(\tau) v_{l}(\tau) d \tau, \hat{E}_{e}=\int_{0}^{t} \hat{F}_{e}(\tau) v_{l}(\tau) d \tau
$$

where the $\hat{E}_{s}$ and $\hat{E}_{e}$ are the observed energy at the spring port and the load port, and $\hat{F}_{e}$ is the estimated external force utilizing the force observer proposed in [27].

$\mathrm{PC}$ is designed to preserve the passivity in real-time by forcing the system to dissipate the monitored energy when the system is in violation of the passivity condition. The energy measured by the PO is generally expressed in discrete time as follows [20]:

$$
E(n)=\Delta T \sum_{i=0}^{n} f(n) v(n)=E(n-1)+\Delta T f(n) v(n)
$$

where $\Delta T$ is the sampling period. Note that $f(n)$ and $v(n)$ are defined as the estimated external force $\hat{F}_{e}$ and the load velocity $v_{l}$ for SEA at the load port.

Based on this measured energy, PC modifies the original force $f$ into the $\mathrm{PC}$ force $f^{\mathrm{PC}}$ as follows:

$$
\begin{aligned}
\alpha(n) & = \begin{cases}-E(n) /\left\{\Delta T v(n)^{2}\right\}, & \text { if } E(n)<0 \\
0, & \text { if } E(n) \geq 0\end{cases} \\
f^{\mathrm{PC}}(n+1) & =f(n+1)+\alpha(n) v(n) .
\end{aligned}
$$

The condition in (13) implies that the PC only intervenes when the measured energy is negative, namely, when the passivity is violated.

The new PC force $f^{\mathrm{PC}}$ is calculated at the next step by adding the product of the previous $\alpha(n)$ and the velocity $v(n)$ to the original force $f(n+1)$. Then, this new control input/force $f^{\mathrm{PC}}$ works to dissipate the violated energy $E(n)$ and thus remove this term from the generated energy at the next step $E(n+1)$.

\section{B. Limitation of PC for Rendering Desired Damping}

Note that the modified term $\alpha(n) v(n)$ can be considered as additional damping, with the damping coefficient $\alpha$ appropriately designed. Therefore, it is natural to come up with the idea that the rendered damping $D_{\text {des }}$ in the desired impedance model (6) can be utilized as PC dissipative term. However, this approach cannot properly modulate the energy dissipation, and thus cannot increase the maximum achievable stiffness.
The limitation of this approach, PC design utilizing the desired impedance model (6) can be explained by the phase characteristic of the rendered impedance. Fig. 8 shows the bode diagram of impedance at the load port with several different $D_{\text {des }}$ values. It can be verified that $D_{\text {des }}$ can affect the phase characteristics but they still deviate over $90^{\circ}$ even when the desired damping increases. In other words, the passivity condition cannot be recovered through the increase of $D_{\text {des. }}$. The cause of these consequences can be confirmed through mathematical analysis.

The real part of the impedance including the desired damping term is derived as follows:

$$
\begin{aligned}
\Re\left(Z_{s}(i \omega)\right)= & \frac{N B_{m} K\left(N K-K_{d e s} \Lambda_{p}+K \Lambda_{p}\right)}{\left(B_{m} \omega\right)^{2}+\left(N K \Lambda_{p}+N^{2} K-J_{m} \omega^{2}\right)^{2}} \\
& +\frac{D_{\text {des }} N K \Lambda_{p}\left(N K \Lambda_{p}+N^{2} K-J_{m} \omega^{2}\right)}{\left(B_{m} \omega\right)^{2}+\left(N K \Lambda_{p}+N^{2} K-J_{m} \omega^{2}\right)^{2}} .
\end{aligned}
$$

The real part consists of two terms: the first term has only one negative component related to the desired stiffness, $-K_{\text {des }}$, where its positiveness can be guaranteed when $K_{\mathrm{des}}$ is lesser than $K$, the inherent stiffness. The second term which is related to the desired damping $D_{\text {des }}$ seems to add positive values to the whole real part and thus contribute to the passivity of the system.

However, the positiveness of the second term is limited only in the low frequency range because of the term $-J_{m} \omega^{2}$, and it becomes negative when the frequency $\omega$ is higher than a certain level. The specific frequency is given as follows:

$$
\omega_{c}=\sqrt{\frac{N K \Lambda_{p}+N^{2} K}{J_{m}}} .
$$

This frequency is where the phase characteristic of the inner force control closed loop (from the spring force reference to the spring force output) becomes $-90^{\circ}$, which varies with respect to the inner force gain $\Lambda_{p}$. However, the second term of (15) becomes negative after this frequency, and the desired damping $D_{\text {des }}$ is unable to guarantee the energy recovery and passivity.

This limitation can be attributed to the fact that the desired load impedance is realized through the spring force control, which means that the desired load damping is not directly added to the load side, but realized through the spring force control.

\section{Design of PC Based on Load Damping Control}

As aforementioned, it is verified that the PC approach utilizing the reference impedance rendering cannot solve the passivity violation. To address this issue, it is necessary to develop a new control method which can directly apply the damping to the desired port, not through the spring force reference.

In order to render the damping directly at the load port, both the dynamic model and passivity have to be taken into account in the controller design. Recently, the elastic structure preserving (ESP) control has been developed to passively add the desired force on the load side [28]-[30]. The ESP preserves the intrinsic inertia and spring properties of the system while adding the desired force on the load side.

The basic idea of ESP control is to add the desired force directly to the load side, such that it can affect the load-side 
dynamics while preserving the structure of SEA, i.e., the spring stiffness and the inertia of the motor and the load. For rendering the desired dynamics at the load side, the motor torque input is designed as

$$
\tau_{m}=\bar{u}+\frac{J_{m}^{n}}{N K} \ddot{\beta}+\frac{B_{m}^{n}}{N K} \dot{\beta}+N \beta
$$

where $J_{m}^{n}$ and $B_{m}^{n}$ are the nominal model parameters of the motor dynamics and $\beta$ is a new coordinate defined to represent the desired force of the load side that will be realized by the control input $\tau_{m}$.

In this article, the desired load-side force $\beta$ is designed to implement the PC force, which is the product of the desired damping calculated by the PC $D_{\mathrm{des}}^{\mathrm{PC}}$ and the load velocity $\dot{x}_{l}$, expressed as follows:

$$
\beta=-D_{\mathrm{des}}^{\mathrm{PC}} \dot{x}_{l}
$$

Then, the resulting closed-loop dynamics is determined from the SEA dynamics (1)-(3) as

$$
\begin{aligned}
M \ddot{x}_{l}+B_{l} \dot{x}_{l}+D_{\mathrm{des}}^{\mathrm{PC}} \dot{x}_{l} & =F_{s}^{\eta}+F_{e} \\
J_{m} \ddot{\eta}+B_{m} \dot{\eta}+N F_{s}^{\eta} & =\bar{u}, \\
F_{s}^{\eta} & =K\left(N \eta-x_{l}\right),
\end{aligned}
$$

where $\eta=\theta-K^{-1} \beta$ is the new motor coordinate. The final closed-loop dynamics from (19) to (21) shows that the desired damping is successfully attached to the load side, whereas preserving the SEA structure.

The proposed PC is designed based on the ESP control concept, and the following theorem verifies its passivity:

Theorem 1: Assume that the SEA dynamics are expressed as (1)-(3).

Let the proposed controller be defined as (17) and (18). Then, the controlled SEA satisfies the following passivity condition at the load port:

$$
\Delta T \sum_{i=0}^{n} F_{e}(i) \dot{x}_{l}(i) \geq 0
$$

Proof: The energy at a certain time $t$ is given as

$$
E(t)=\Delta T \sum_{i=0}^{n} F_{e}(i) \dot{x}_{l}(i)
$$

By the proposed PC, the force $F_{e}(t)$ is modulated to $\tilde{F}_{e}(t)$ as

$$
\tilde{F}_{e}(t)=F_{e}(t)+D_{\text {des }}^{\mathrm{PC}} \dot{x}_{l}(t) \text { if } E(t)<0 .
$$

The desired damping value is designed as

$$
D_{\mathrm{des}}^{\mathrm{PC}}=-\frac{E(t)}{\left(\Delta T \dot{x}_{l}\right)^{2}}
$$

which modulate the energy consumption to be zero as follows:

$$
\begin{aligned}
\tilde{E}(t) & =\Delta T \sum_{i=0}^{n} F_{e}(i) \dot{x}_{l}(i)+\Delta T D_{\mathrm{des}}^{\mathrm{PC}}(t) \dot{x}_{l}(t) . \\
& =E(t)-E(t)=0 .
\end{aligned}
$$

Therefore, the updated energy satisfies the passivity condition.

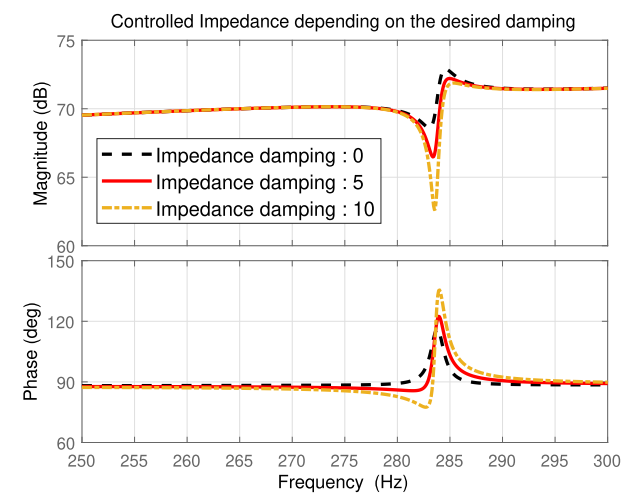

Fig. 8. Impedance at the load port according to the desired damping.

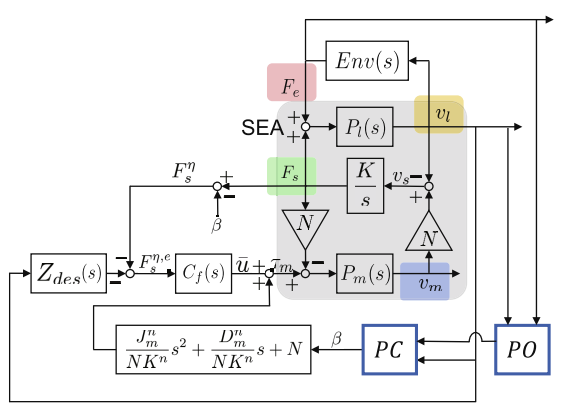

(a)

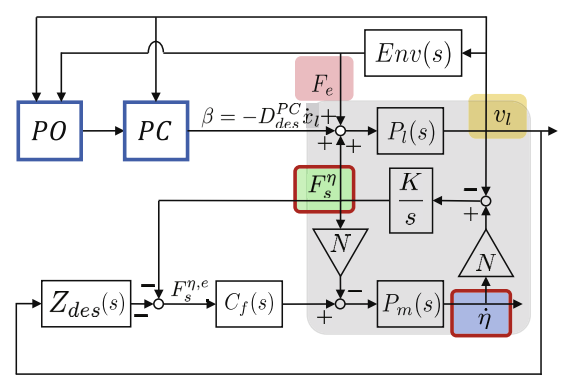

(b)

Fig. 9. Block diagrams. (a) PC. (b) Equivalent structure. $F_{s}^{\eta, e}$ indicates error of spring force in the transformed coordinate.

The controlled impedance at the load port can be derived in the $S$-domain after applying the PC, and its real part is determined as follows:

$\Re\left(Z_{l}^{\mathrm{PC}}\right)=D_{\mathrm{des}}^{\mathrm{PC}}+B_{l}+\frac{N B_{m} K\left(N K-K_{\mathrm{des}} \Lambda_{p}+K \Lambda_{p}\right)}{\left(B_{m} \omega\right)^{2}+\left(N K \Lambda_{p}+N^{2} K-J_{m} \omega^{2}\right)^{2}}$.

It can be verified that the desired damping $D_{\mathrm{des}}^{\mathrm{PC}}$ is separately added to the real part of the original impedance, and thus can successfully compensate for the positiveness. Accordingly, the passivity of the system can be maintained by controlling $D_{\text {des }}^{\mathrm{PC}}$ with PC.

Overall control structure of the proposed passivity observer and passivity controller (POPC) for SEA is configured as in Fig. 9. PO at the load port is designed as (12) in Section III-A, using the load velocity and the estimated external force, and PC is triggered by PO as shown in (13) and (14). In the proposed 


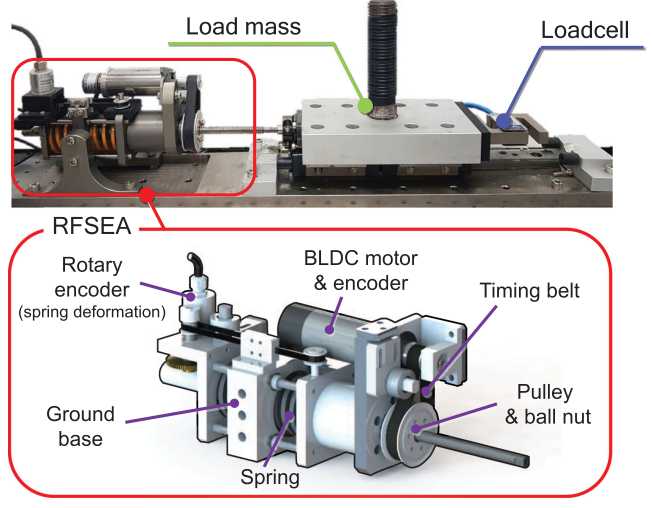

Fig. 10. Experiment set-up consists of RFSEA [31], load mass and load cell.

TABLE IV

PARAMETERS UtILIZED IN THE EXPERIMENTS

\begin{tabular}{cll}
\hline \hline Identified Parameter & Description & Value \\
\hline$J_{m}$ & Motor inertia & $0.64 \mathrm{e}-5 \mathrm{~kg} \cdot \mathrm{m}^{2}$ \\
$B_{m}$ & Motor damping & $0.6 \mathrm{e}-4 \mathrm{Nm} \cdot \mathrm{s} / \mathrm{rad}$ \\
$K$ & Spring stiffness & $160 \mathrm{kN} / \mathrm{m}$ \\
$M_{l}$ & Load mass & $1.9 \mathrm{~kg}$ \\
$B_{l}$ & Load damping & $100 \mathrm{~N} \cdot \mathrm{s} / \mathrm{m}$ \\
\hline \hline Controller Parameter & Description & Value \\
\hline$K_{d e s}$ & Desired stiffness & $0.5 K \sim 7 K$ \\
$\Lambda_{p}$ & Inner force P gain & $1 \mathrm{e}-3 / 1 \mathrm{e}-1$ \\
$\Lambda_{d}$ & Inner force D gain & $1 \mathrm{e}-4$ \\
$Q$ & Cut-off frequency of filter & $150 \mathrm{~Hz}$ \\
\hline \hline Measured / Estimated & Description \\
Parameter & \\
\hline$v_{l}$ & Load velocity \\
$F_{s}$ & Spring force & \\
$F_{s}^{\eta}$ & Spring force in the transformed coordinate \\
$\hat{F}_{e}$ & Estimated external force \\
\hline
\end{tabular}

POPC, $D_{\text {des }}^{\mathrm{PC}}$ in (18) is employed as $\alpha$ in (13), and it is added by $\beta$ to the motor input in (17) as shown in Fig. 9(a). The equivalent block diagram can be rewritten according to (19) to (21) as shown in Fig. 9(b), where the desired damping $D_{\mathrm{des}}^{\mathrm{PC}}$ is directly added to the load port. The velocity $\dot{\eta}$ and the spring force $F_{s}^{\eta}$ in the transformed coordinate are marked by the red boxes in Fig. 9(b), where the structure of the SEA is preserved.

In brief, this section proposes the new POPC algorithm for SEA taking into consideration the passivity analysis proposed in Section II. The system parameters required to design the proposed POPC can be obtained by utilizing system identification methods such as the frequency response function measurement. The detailed and theoretical analysis is to be conducted as the future work.

\section{EXPERIMENTS}

Experiments are conducted to validate the rendering performance of the desired stiffness and verify the effectiveness of the proposed POPC for SEA. Fig. 10 illustrates the SEA system that is utilized for the experiments. Reaction force-sensing SEA (RFSEA) [31], [32] with load masses over the linear motion

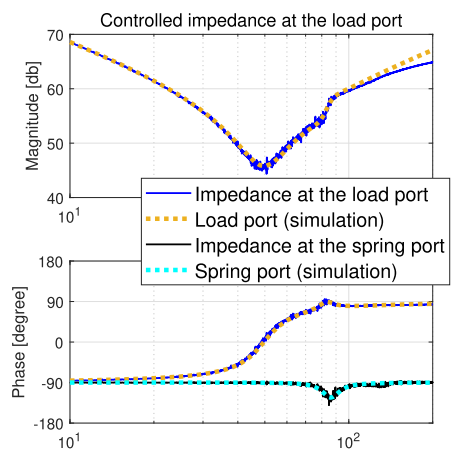

(a)
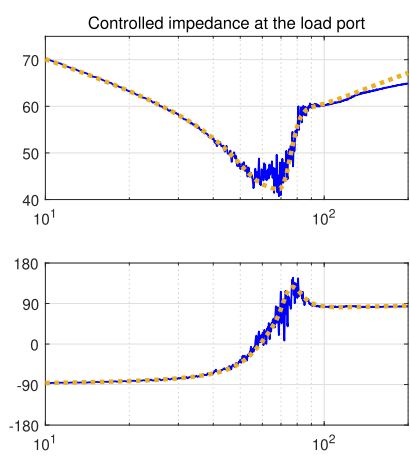

(b)
Fig. 11. Frequency characteristics of the controlled impedance when the desired stiffness is set. (a) $K_{\text {des }}=1.1 \mathrm{~K}$. (b) $K_{\text {des }}=1.3 \mathrm{~K}$ ).

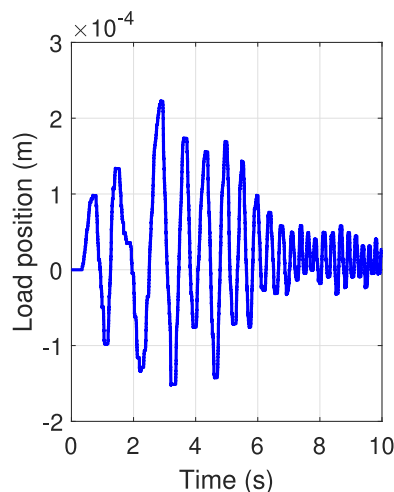

(a)

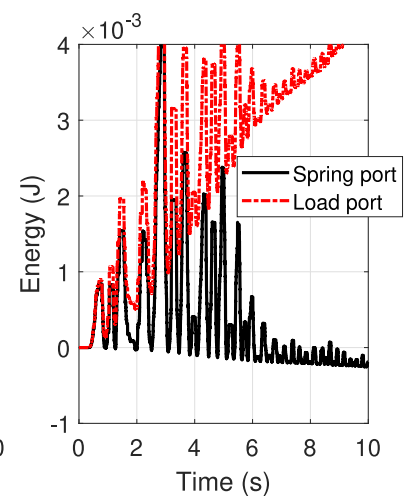

(b)
Fig. 12. Experimental results. (a) Load velocity. (b) Energy when the higher desired stiffness is $\operatorname{set}\left(K_{\text {des }}=1.1 \mathrm{~K}\right)$.

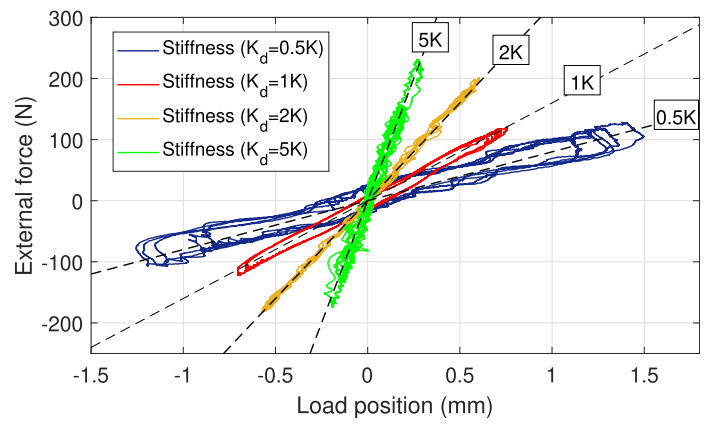

Fig. 13. Rendering performance of various desired stiffness.

guide (LM guide) is adopted as the SEA system to be impedance controlled.

At first, the passivity discussion in Section II-B and II-C is verified through experiments to show the advantage of the load port. Fig. 11 shows the frequency characteristic of the controlled impedance at both the energy ports when the desired stiffness of impedance is set higher than the spring stiffness without the POPC. The blue line indicates the impedance at the load port, and the black line indicates the impedance at the spring port. Yellow and cyan dashed lines show the simulation results. The 


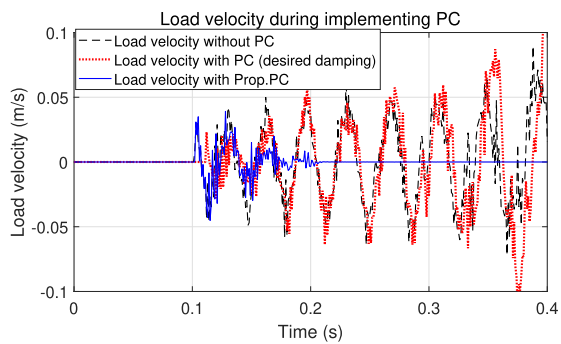

(a)

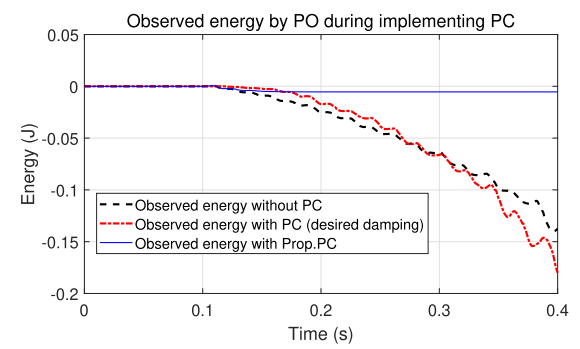

(b)

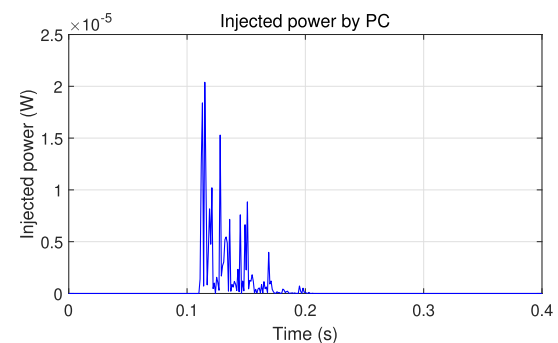

(c)

Fig. 14. (a) Load velocity. (b) Energy when the PC is implemented. (c) Injected power by the PC ( $\left.K_{\text {des }}=7 K\right)$.

load was excited manually, and the inner force $\mathrm{P}$-gain and D-gain are set to $1 \mathrm{e}-1$ and $1 \mathrm{e}-4$.

The result shows that passivity is violated at approximately $80 \mathrm{~Hz}$ at the spring port, while it is preserved at the load port with the phase staying within \pm 90 degrees. Both results are consistent with the simulation results. The experiment to show the passivity violation is also conducted when the desired stiffness is more increased. The passivity can be violated even at the load port when the desired stiffness is set higher, and the experimental results are shown in Fig. 11(b), where the passivity is violated at approximately $80 \mathrm{~Hz}$ at the load port, too.

Fig. 12 illustrates the energy observation comparison result between the $\mathrm{PO}$ at the spring port and the PO at the load port. The system is coupled stable showing that load velocity eventually converges to zero as shown in Fig. 12(b). However, the observed energy in Fig. 12(b) shows the different results depending on the energy port. The energy observed by the PO at the load port remains positive, whereas the energy at spring port PO vastly falls in the negative direction indicating the system is not passive nor coupled stable. This result leads to the conclusion that the load port is the less conservative energy port than the spring port when it comes to monitoring the passivity.

\section{A. Impedance Rendering of Various Desired Stiffness}

The experiment is designed to validate the rendering performance of the desired stiffness $K_{\text {des }}$ utilizing the analysis in Section II, which is examined by calculating the impedance between the load position $x_{l}$ and the measured external force $F_{e}^{\text {measured }}$. For these experiments, the load cell was used on the load part for more accurate measurement of the external force (CAS, SBA-50). The external forces in both directions were applied manually to the load cell. Fig. 13 shows the comparison between the desired stiffness and the measured stiffness. The results indicate that the various conditions of the stiffness are successfully rendered.

\section{B. Experimental Validation of POPC for SEA}

For the validation of the POPC in the time-domain, The desired stiffness, $K_{\text {des }}=1120 \mathrm{kN} / \mathrm{m}$, is set seven times higher than the spring stiffness as the desired impedance, and impulse forces are applied by a hammer to the load-side mass. The inner force P-gain and D-gain are set to $1 \mathrm{e}-3$ and $1 \mathrm{e}-4$ which are set as high as possible while avoiding motor saturation.
The result verifies that only the proposed PC can stabilize the system regulating the velocity shown in Fig. 14(a). The energy observation elaborates on the advantages of the proposed PC in detail; the observed energy in Fig. 14(b) indicates that the system without the PC and with the PC (by impedance damping) becomes nonpassive showing negative energy dissipation. On the other hand, the energy with the proposed POPC converges and preserves passivity. Fig. 14(c) shows that the proposed PC can inject the energy to recover the passivity and it converges to zero after the velocity converges to zero.

It is true that a small error of energy remains in the proposed POPC case, but this is because the controlled damping cannot be exactly the same as the real damping when a collision occurred. The nonlinearity such as friction, modeling uncertainty, and saturation can affect the performance in the experiments, but the system under the proposed PC can maintain its stability regardless of these factors.

This result verifies that the proposed POPC can engage the load side dynamics after the measured energy drops below zero and recover the passivity by applying additional damping to the load side. The preserved passivity leads to the stability of the system, too; the load velocity becomes unstable without the proposed POPC in Fig. 14(a), since the system is not passive, while the load velocity with the proposed POPC decreases after the $\mathrm{PC}$ intervenes and eventually recovers its stability.

\section{CONCLUSION}

This article analyzed the passivity of SEA under impedance control based on the model and its frequency response analysis, and the novel PC algorithm was designed based on the analysis. Consequently, the achievable stiffness could be considerably increased beyond the conventional theoretical limit, which was a well-recognized challenge in impedance-controlled SEA. It thus fosters wider applicability of the SEA.

Based on the precise model of SEA, it was shown that the passivity analysis at the load port was less conservative than that at the spring port, and thus the desired stiffness for SEA impedance control could be set higher than the intrinsic spring stiffness. Moreover, the condition of parameters to maximize the desired stiffness was examined by analyzing the relationship between parameters and the positive realness of the impedance at the load port. In conclusion, we confirmed that D-gain of the force control and the load damping played a significant role in 
relaxing the passivity, whereas the P-gain of the force control could not relax the passivity.

The time-domain POPC algorithm was designed taking into consideration the dynamic characteristics of SEA to preserve the passivity of SEA.

Experiments verified the less conservative characteristic of the load port, and it was validated that the proposed POPC could increase the maximum value of the rendered stiffness. As the proposed POPC relies on the linear model, the robustness of the proposed algorithm against various nonlinear factors including friction, modeling uncertainty should be investigated in the future work.

\section{REFERENCES}

[1] G. A. Pratt and M. M. Williamson, "Series elastic actuators," in Proc. IEEE/RSJ Int. Conf. Intell. Robots Syst. Human Robot Interaction Cooperative Robots, 1995, vol. 1, pp. 399-406.

[2] J. Pratt, B. Krupp, and C. Morse, "Series elastic actuators for high fidelity force control," Ind. Robot, Int. J., vol. 29, no. 3, pp. 234-241, 2002.

[3] S. Oh and K. Kong, "High-precision robust force control of a series elastic actuator," IEEE/ASME Trans. Mechatronics, vol. 22, no. 1, pp. 71-80, Feb. 2017.

[4] H. Lee and S. Oh, "Design of reduced order disturbance observer of series elastic actuator for robust force control," in Proc. IEEE 15th Int. Workshop Adv. Motion Control, 2018, pp. 663-668.

[5] E. Sariyildiz, H. Yu, T. Nozaki, and T. Murakami, "Robust force control of series elastic actuators using sliding mode control and disturbance observer," in Proc. 42nd Аnnu. Conf. IEEE Ind. Electron. Soc., 2016, pp. 619-624.

[6] A. Calanca and P. Fiorini, "A rationale for acceleration feedback in force control of series elastic actuators," IEEE Trans. Robot., vol. 34, no. 1, pp. 48-61, Feb. 2018.

[7] N. Paine et al., "Actuator control for the nasa-jsc valkyrie humanoid robot: A decoupled dynamics approach for torque control of series elastic robots," J. Field Robot., vol. 32, no. 3, pp. 378-396, 2015.

[8] Y. Zhao, N. Paine, S. J. Jorgensen, and L. Sentis, "Impedance control and performance measure of series elastic actuators," IEEE Trans. Ind. Electron., vol. 65, no. 3, pp. 2817-2827, Mar. 2018.

[9] M. A. Hopkins, S. A. Ressler, D. F. Lahr, A. Leonessa, and D. W. Hong, "Embedded joint-space control of a series elastic humanoid," in Proc. IEEE/RSJ Int. Conf. Intell. Robots Syst., 2015, pp. 3358-3365.

[10] J. Lee et al., "Upper-body impedance control with variable stiffness for a door opening task," in Proc. 14th IEEE-RAS Int. Conf. Humanoid Robots, 2014, pp. 713-719.

[11] S. Wang et al., "Design and control of the MINDWALKER exoskeleton," IEEE Trans. Neural Syst. Rehabil. Eng., vol. 23, no. 2, pp. 277-286, Mar. 2015.

[12] H. K. Kwa, J. H. Noorden, M. Missel, T. Craig, J. E. Pratt, and P. D. Neuhaus, "Development of the IHMC mobility assist exoskeleton," in Proc. IEEE Int. Conf. Robot. Autom., 2009, pp. 2556-2562.

[13] H. Lee, S. Kwak, and S. Oh, "Force control of series elastic actuatorsdriven parallel robot," in Proc. IEEE Int. Conf. Robot. Autom., May 2018, pp. $1-5$.

[14] J. E. Colgate, "The control dynamically interacting systems," Ph.D. thesis, Dept. Mech. Eng., Massachusetts Inst. Technol., Cambridge, MA, USA, 1988.

[15] H. Vallery, J. Veneman, E. Van Asseldonk, R. Ekkelenkamp, M. Buss, and H. Van Der Kooij, "Compliant actuation of rehabilitation robots," IEEE Robot. Autom. Mag., vol. 15, no. 3, pp. 60-69, Sep. 2008.

[16] N. L. Tagliamonte and D. Accoto, "Passivity constraints for the impedance control of series elastic actuators," Proc. Institution Mech. Engineers, Part I, J. Syst. Control Eng., vol. 228, no. 3, pp. 138-153, 2014.

[17] A. Calanca, R. Muradore, and P. Fiorini, "Impedance control of series elastic actuators: Passivity and acceleration-based control," Mechatronics, vol. 47, pp. 37-48, 2017.

[18] D. P. Losey and M. K. O'Malley, "Effects of discretization on the k-width of series elastic actuators," in Proc. IEEE Int. Conf. Robot. Autom., 2017, pp. 421-426.
[19] H. Lee, J. Lee, J. Ryu, and S. Oh, "Relaxing the conservatism of passivity condition for impedance controlled series elastic actuators," in Proc. IEEE/RSJ Int. Conf. Intell. Robots Syst., 2019, pp. 7610-7615.

[20] B. Hannaford and J.-H. Ryu, "Time-domain passivity control of haptic interfaces," IEEE Trans. Robot. Autom., vol. 18, no. 1, pp. 1-10, Feb. 2002.

[21] J.-H. Ryu, D.-S. Kwon, and B. Hannaford, "Control of a flexible manipulator with noncollocated feedback: Time domain passivity approach," Control Problems Robot., vol. 4, pp. 121-134, 2003.

[22] J.-H. Ryu, C. Preusche, B. Hannaford, and G. Hirzinger, "Time domain passivity control with reference energy following," IEEE Trans. Control Syst. Technol., vol. 13, no. 5, pp. 737-742, Sep. 2005.

[23] D. P. Losey, A. Erwin, C. G. McDonald, F. Sergi, and M. K. O’Malley, "A time-domain approach to control of series elastic actuators: Adaptive torque and passivity-based impedance control," IEEE/ASME Trans. Mechatronics, vol. 21, no. 4, pp. 2085-2096, Aug. 2016.

[24] H. Yu, S. Huang, G. Chen, Y. Pan, and Z. Guo, "Human-robot interaction control of rehabilitation robots with series elastic actuators," IEEE Trans. Robot., vol. 31, no. 5, pp. 1089-1100, Oct. 2015.

[25] M. Mosadeghzad, G. A. Medrano-Cerda, J. A. Saglia, N. G. Tsagarakis, and D. G. Caldwell, "Comparison of various active impedance control approaches, modeling, implementation, passivity, stability and tradeoffs," in Proc. IEEE/ASME Int. Conf. Adv. Intell. Mechatronics, 2012, pp. $342-348$.

[26] F. Sergi and M. K. O'Malley, "On the stability and accuracy of high stiffness rendering in non-backdrivable actuators through series elasticity," Mechatronics, vol. 26, pp. 64-75, 2015.

[27] Y. Park, N. Paine, and S. Oh, "Development of force observer in series elastic actuator for dynamic control," IEEE Trans. Ind. Electron., vol. 65, no. 3, pp. 2398-2407, Mar. 2018

[28] M. Keppler, D. Lakatos, C. Ott, and A. Albu-Schäffer, "A passivitybased approach for trajectory tracking and link-side damping of compliantly actuated robots," in Proc. IEEE Int. Conf. Robot.Autom., 2016, pp. 1079-1086.

[29] M. Keppler, D. Lakatos, C. Ott, and A. Albu-Schaffer, "Elastic structure preserving impedance (es $\pi$ ) control for compliantly actuated robots," in Proc. IEEE/RSJ Int. Conf. Intell. Robots Syst., 2018, pp. 5861-5868.

[30] M. Keppler, D. Lakatos, C. Ott, and A. Albu-Schäffer, "Elastic structure preserving (ESP) control for compliantly actuated robots," IEEE Trans. Robot., vol. 34, no. 2, pp. 317-335, Apr. 2018.

[31] N. Paine, S. Oh, and L. Sentis, "Design and control considerations for highperformance series elastic actuators," IEEE/ASME Trans. Mechatronics, vol. 19, no. 3, pp. 1080-1091, Jun. 2014.

[32] C. Lee, S. Kwak, J. Kwak, and S. Oh, "Generalization of series elastic actuator configurations and dynamic behavior comparison," Actuators, vol. 6 , no. 3, 2017, Art. no. 26.

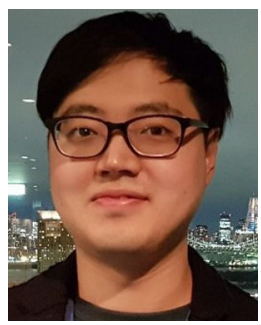

Hyunwook Lee (Student Member, IEEE) received the B.S. degree in mechanical engineering from Pusan National University, Pusan, South Korea, in 2014 and the M.S. degree in robotics engineering in 2016 from the Daegu Gyeongbuk Institute of Science and Technology (DGIST), Daegu, where he is currently working toward the Ph.D. degree in series elastic actuators-driven parallel robot for dynamic human interaction.

His current research interests include humanrobot interaction applications, development of motion control algorithms for compliant robot.

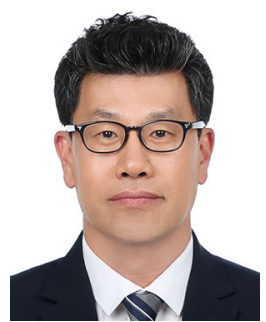

Jee-Hwan Ryu (Senior Member, IEEE) received the B.S. degree from Inha University, Incheon, South Korea, in 1995, and the M.S. and Ph.D. degrees from the Korea Advanced Institute of Science and Technology, Taejon, in 1997 and 2002, respectively, all in mechanical engineering.

$\mathrm{He}$ is currently an Associate Professor with the Department of Civil and Environmental Engineering, Korea Advanced Institute of Science and Technology. His research interests include haptics, telerobotics, teleoperation, exoskeletons, and autonomous vehicles. 


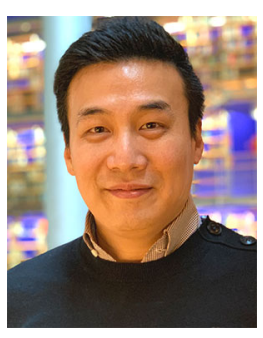

Jinoh Lee (Senior Member, IEEE) received the B.S. degree from Hanyang University, Seoul, South Korea, in 2003 (awarded Summa Cum Laude), and the M.Sc. and Ph.D. degrees from the Korea Advanced Institute of Science and Technology, Daejeon, in 2012, all in mechanical engineering.

He held postdoctoral position from 2012 to 2017 and Research Scientist position from 2017 to 2020 with the Department of Advanced Robotics, Istituto Italiano di Tecnologia, Genoa, Italy. He is currently a Research Scientist with the Institute of Robotics and Mechatronics Center, German Aerospace Center (DLR), Weling, Germany. His professional is about robotics and control engineering, which include manipulation of highly redundant robots such as dual-arm and humanoids, robust control of nonlinear systems, and compliant robotic system control for safe human-robot interaction.

Dr. Lee is a member of the IEEE Robotics and Automation, the IEEE Control Systems, and the IEEE Industrial Electronics Societies.

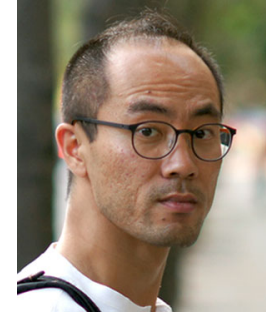

Sehoon Oh (Senior Member, IEEE) received the B.S., M.S., and Ph.D. degrees in electrical engineering from The University of Tokyo, Tokyo, Japan, in 1998, 2000, and 2005 respectively.

He was a Research Associate with The University of Tokyo until 2012, a Visiting Researcher with the University of Texas at Austin from 2010 to 2011 . He is currently an Associate Professor with the Department of Robotics Engineering at DGIST, Daegu, South Korea. $\mathrm{He}$ has been working on many projects regarding advanced human machine interaction particularly focusing on design of actuators and controllers.

Dr. Oh was the recipient of the Best Transactions Paper Award from the IEEE TRANSACTIONS ON INDUSTRIAL ELECTRONICS in 2013. 